American Journal of Animal and Veterinary Sciences 3 (2): 68-72, 2008

ISSN 1557-4555

(C) 2008 Science Publications

\title{
Corynebacterium pseudotuberculosis Infection (Caseous Lymphadenitis) in Camels (Camelus dromedarius) in Jordan
}

\author{
Azmi Dawood Hawari \\ Department of Nutrition and Food Processing, Faculty of Agricultural Technology, \\ Al-Balqa Applied University, Al-Salt 19117, Jordan
}

\begin{abstract}
Problem statement: This study was conducted to describe \& report for the first time outbreaks of natural C.pseudotuberculosis infection in adult camel herds (Camelus dromedarius) in Jordan. An infectious disease syndrome was reported in three camel herds (Camelus dromedarius) intensively raised at south province in Jordan. Approach: The herds included over 160 adult camels out of which about $8 \%$ were affected with multiple muscle and subcutaneous abscesses at various sites of the body. The camels were also heavily infested with ticks. Results: The infected camels did not respond favorably to several broad spectrum antibiotics. Post-mortem examination of 5 carcasses revealed emaciation and presence of external and internal multiple abscesses particularly in the lungs. The abscesses were encapsulated by fibrous tissue and contained creamy yellowish white pus. The lymph nodes were slightly congested and swollen. Conclusion: Corynebacterium pseudotuberculosis type I strain or biovar ovis (the known cause of caseous lymphadenitis in sheep) was isolated from pus, lymph nodes, ticks, milk, blood and liver samples. The clinical symptoms, nature and distribution of lesions of caseous lymphadenitis in camels are not as typical as in sheep. Recommendations for pseudotuberculosis control were given.
\end{abstract}

Key words: Caseous lymphadenitis in camel, Corynebacterium pseudotuberculosis

\section{INTRODUCITON}

Corynebacterium pseudotuberculosis is a small club-shaped rod (1-2 $\mu \mathrm{m})$ which produces lesions similar to those of tuberculosis. It is known world-wide to cause pseudotuberculosis or caseous lymphadentitis in adult sheep. Usually the large superficial lymph nodes are affected but occasionally abscessation of the nodes of the internal organs may also occur. Severe economic losses result from unthriftiness and death of some sheep and from condemnation of infected carcasses at slaughter. Furthermore, the infection of goats, deer, horses (Ulcerative lymphangitis), cattle and man adds both economic importance and public health significance to the malady ${ }^{[1,2,3]}$.

On the other hand, some reports on $C$. pseudotuberculosis infection in camels were recorded. In Saudi Arabia ${ }^{[4]}$ isolated C. pseudotuberculosis strain from eight camels with caseous lymphadenitis. Postmortem revealed emaciation and presence of multiple external and internal abscesses particularly in the lungs and liver. The body lymph nodes were enlarged and congested but without abscesses formation. In Ethiopia $^{[5]}$ reported a chronic condition locally called mala in adult camels which resembled caseous lymphadenitis in small ruminants. In certain herds up to
$15 \%$ of adult camels were found affected at one time. When the isolated $C$. pseudotuberculosis and group $B$ streptococci were inoculated intravenously in a camel, they produced polyarthritis and numerous muscle and subcutaneous abscesses. In United Arab Emirates ${ }^{[6]}$ isolated C. pseudotuberculosis from eleven cases of lymphadenitis in camel.

The purpose of this study is to report and describe for the first time outbreaks of natural $\mathrm{C}$. pseudotuberculosis infection in adult camels (Camelus dromedarius) in Jordan.

\section{MATERIALS AND METHODS}

Herd history and clinical symptoms: The investigation was performed on three camel herds of about 160 adult camels of both sexes, intensively raised at south province of Jordan near Saudi Arabia border. Two herds of camels which were originally purchased from different regions in Saudi Arabia. In the third herd, the majority of camels were locally purchased. From May to November 2005 about $10 \%$ of the camels in both herds developed the following clinical symptoms: appearance of numerous muscle and subcutaneous abscesses of various sizes particularly at the hind-quarters, shoulders, base of the neck, under the jaw (Fig. 1) and at the base of the tail. 


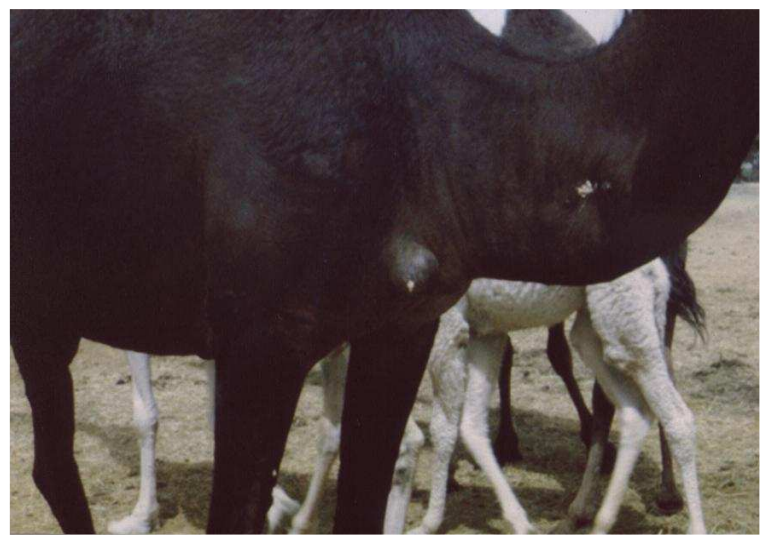

Fig. 1: Muscle and subcutaneous abscesses caused by C. pseudotuberculosis

Table 1: Specimens from affected camels

\begin{tabular}{lll}
\hline Kind of Specimens & Total (No.) & Source of specimens \\
\hline Pus & $(22)$ & $\begin{array}{l}\text { Abscesses from lunges, } \\
\text { liver, joints, muscular and } \\
\text { subcutaneous tissues of thigh, } \\
\text { axilla, base of tail, base of } \\
\text { neck, shoulder, elbow and } \\
\text { under jaw. }\end{array}$ \\
Lymph nodes & (10) & $\begin{array}{l}\text { Prescapular, precrural, } \\
\text { bronchial, } \\
\text { mesenteric and mediastinal. }\end{array}$ \\
Ticks & & $\begin{array}{l}\text { From camels with multiple } \\
\text { abscesses. }\end{array}$ \\
Milk & $(12)$ & $\begin{array}{l}\text { From camels with multiple } \\
\text { abscesses. }\end{array}$ \\
Blood & $(5)$ & From affected camels. \\
Liver & From camel carcasses at P.M. \\
Intestinal contents & $(4)$ & From alive and dead camels. \\
\hline
\end{tabular}

Some camels were unable to walk normally due to the presence of abscesses at the joints of the limbs. Some abscesses had sinus tracts draining purulent materials to the outside. In few affected camels the peripheral lymph nodes were slightly enlarged but without abscess formation. In one herd, only five camels died over three to four months. Theses camels showed progressive weakness, difficult breathing, paleness of mucous membranes, diarrhea, loss of appetite, emaciation and depression. In both herds, the entire body of most of the camels was heavily infested with ticks of Hyaloma species. The infected camels did not respond favorably to repeated inoculation with several broad spectrum antibiotics and anthelmintics (Neodiarestin, Oxytetracycline, Thiabenzole, Ivomec and Naganol).

Post-mortem examination: Immediately after death, post-mortem examination was carried out. The following post-mortem lesions were observed: Severe emaciation and absence of internal body fat. The body lymph nodes were slightly enlarged and congested but without abscesses formation. Numerous abscesses of various sizes (from barely visible to as large as orange size) were found in the muscles and subcutaneous tissues of the thigh, shoulder, elbow, base of the neck, axilla region, under the jaw and on the joints. Multiple large abscesses were also found in internal organs particularly the lungs. In two out of 5 carcasses, the joints of the four limbs contained abscesses. The abscesses were encapsulated by a relatively thick layer of necrotic and fibrous tissues. They contained odorless, non-granular and non-calcified thin creamy yellowish white pus (and sometimes tinged with blood). The liver in two carcasses was pale, friable and contained peticheal hemorrhages. The mucosa of the gastro-intestinal tract was congested.

Specimens and microbiological examinations: the following specimens shown in Table 1 were aseptically collected from affected camels (alive and dead) for microbiological studies: (a) blood smears stained with Giemsa (Ventana) for blood parasites examination, (b) impression smears from mesenteric lymph nodes and from scrapings of intestinal mucosa, stained with Ziehl Neelsen's method for examination for acid-fast bacteria and (c) pus from abscesses in the body, superficial and internal lymph nodes, liver, blood on EDTA, milk, ticks and intestinal contents for bacteriological examination, using standard materials and procedures, Merchant and Packer ${ }^{[5]}$.

Virulence to guinea pigs: Guinea pigs were inoculated intradermally and intraperitoneally with 0.1 and $0.2 \mathrm{~mL}$ of $72 \mathrm{~h}$ broth culture of the organism respectively. The guinea pigs were examined for gross pathology and heart's blood was cultured.

\section{RESULTS}

A Corynebacterium pseudotuberculosis type I strain was isolated on blood agar plates from the following specimens: (a) all the twenty-two pus specimens obtained from abscesses in lungs, liver, joint, muscular and subcutaneous tissues of the thigh, axilla, base of the tail, shoulder, elbow, base of the neck and under the jaw. In addition, Staphylococcus aureus (2 out of 22), C. renali (1 out of 22) and C. equi (1 out of 22) are occasionally isolated from some open abscesses (Table 2), (b) nine out of ten superficial and internal lymph nodes, (c) from ten out of twelve collections of engorged ticks which were obtained from camels affected with multiple abscesses. 
American J. Animal \& Vet. Sci., 3 (2): 68-72, 2008

Table 2: The isolated bacteria from specimens of affected camels

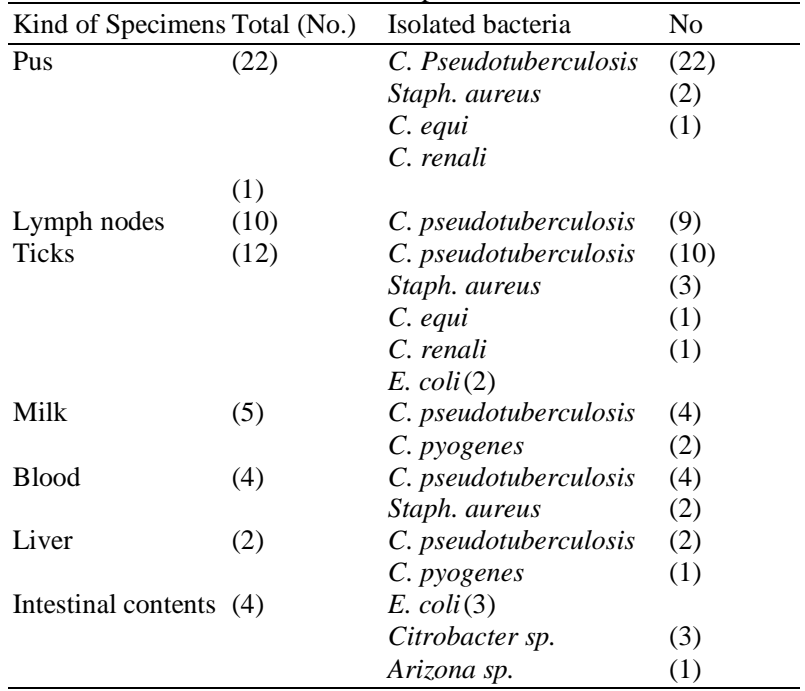

In addition, other bacteria such as Staphlyococcus aureus, (3 out of 12), C. renali (1 out of 12), C. equi (1 out of 12) and E. coli (2 out of 12) were also isolated (Table 2), (d) four out of five milk samples from camels with subcutaneous abscesses, in addition, C.pyogenes ( 2 out of 5) were also isolated (e) four blood specimens from affected camels. Also Staphylococcus aureus (2 out 4) were isolated and from (f) the two liver specimens. Moreover, one isolate of $C$. pyogenes ( 1 out of 2) were isolated (Table 2).

The isolated C. pseudotuberculosis grew clearly after $48 \mathrm{~h}$ of incubation at $37^{\circ} \mathrm{C}$ on heart infusion agar (Oxoid) containing 5\% defibrinated sheep blood. The growth was not improved by incubation in a $\mathrm{CO}_{2}$ atmosphere and relatively good growth was obtained in aerobic conditions. The colonies were rather small though their diameters enlarged up to one $\mathrm{mm}$ after few days of incubation. The isolated strain hydrolysed urea and produced acid from glucose, galactose, maltose,

The in vitro antibiotic susceptibility test on the isolated strain showed complete resistance to Ampicillin, Colistin, Erythromycin, Penicillin and Tetracyclin. However, the test gave moderate sensitivity to Oxytetracyclin, Streptomycin, Doxycyclin and Trimethoprim. It was fully sensitive to Apramycin, Flumiquine, Gentamicin, Neomycin, Linco-spectin, Enrofloxacin, Sulfadiazine and

Trimethoprim+Sulfamethoxazole.

The examination of blood smears from affected camels did not reveal the presence of any blood parasites in spite of the heavy infestation with ticks.

Furthermore, the impression smears prepared from the mesenteric lymph nodes and from the scrapings of the intestinal mucosa of the five dead camels were found free from acid-fast bacteria. However, Arizona sp., E. coli, Shigella and Citrobacter were isolated and identified from the intestinal contents of four affected camels. In addition. Light infestation with Trichostrongylus sp. was found in the feces of one camel.

Animal virulence: The guinea pigs which were inoculated with broth culture of the isolated organism, died three weeks post-inoculation. Post-mortem examination revealed multiple abscesses in the liver and lungs in addition to local abscess at the site of inoculation. Heart's blood and pus were cultured and C. pseudotuberculosis was recovered.mannose and sucrose. It did not ferment arabinose, raffinose, xylose, lactose, rhamnose, inulin, salicin, dulcitol, adonitol and mannitol. The isolated strain did not reduce nitrate, did not produce indol and was negative to the methyl-red and Voges-Proskauer tests. It did not liquify gelatin, coagulated blood serum or milk casein. The culture produced narrow zones of beta hemolysis in the depth of the blood agar medium after $48 \mathrm{~h}$ of incubation at $37^{\circ} \mathrm{C}$.

\section{DISCUSSION}

The literature is very scanty with information about the Arabian camel (Camelus dromedarius) and its diseases. This may be due to the nature of the old camel husbandry and management systems in Arab countries. However, with the recent developments and establishment of several research institutes together with settlement of some large camel herds and initiation of camel herds for commercial milk production there is an increased interest for investigation of major camel problems.

In the present investigation, natural $C$. pseudotuberculosis infection produced a chronic disease (Caseous lymphadenitis) in adult camels which is of economic concern in herds where intensive husbandry was practiced. Clinically and pathologically, the disease in adult camels generally resembled that of sheep with the exception of: (1) the predominance of muscular and subcutaneous abscesses, (2) the lymph nodes were slightly enlarged and congested without development of lamination or caseous necrosis, although they contained C. pseudotuberculosis and (3) the abscesses contained homogenously thin creamy yellowish white pus.

In this study, the predominance of muscular and subcutaneous abscesses and the occasional involvement of the joints and internal organs may be due to the method of transmission of the disease. The isolation of C. pseudotuberculosis from 10 out of 12 collections of engorged ticks and from blood of some infected camels strongly incriminates the ticks in transmission of the disease among camels. However, in a study in Ethiopia $^{[5]}$, intravenous inoculation of a culture of $C$. pseudotuberculosis and group $B$ streptococci produced polyarthritis and numerous muscle and subcutaneous abscesses in one camel. In sheep, it was reported that engorged female ticks, Dermacentor albipictus, 
harbored the organism and guinea pig inoculation with larval ticks reared from such females were also shown to contain $C$. pseudotuberculosis ${ }^{[7]}$. Furthermore, interavenous administratin of $C$. pseudotuberculosis in sheep resulted in polyrthritis and disseminated visceral abscesses mainly in lungs and thoracic lymph nodes ${ }^{[8]}$.

In the present investigation, although $C$. pseudotuberculosis was isolated from 9 out of 10 superficial and internal lymph nodes, there was no detectable caseous necrosis or lamination. However, in a previous investigation in Saudi Arabia on camels it was indication that such a natural $C$. pseudotuberculosis infection resulted in caseous lymphadenitis or cheesy gland disease ${ }^{[4]}$. Furthermore, in some studies camel cases resembled caseous lymphadenitis in small ruminants were described ${ }^{[5,9]}$. They were characterized by the formation of external and internal abscesses, which affected adult camels over five years of age. The lymph glands were involved and most frequently the inferior cervical. Lesions also occurred on the hind quarters and shoulder.

In the present study and also in the one made on Ethiopian camels, the abscesses contained odorless, non-granular, non-calcified thin homogenous creamy yellowish white pus ${ }^{[5]}$. The camel pus was clearly different from that of sheep (with caseous lymphadenitis) which was thick and green in color ${ }^{[1]}$. The difference could be due to the nature of phagocytic enzymes, with those of camels being more liquefactive.

The isolation of C. pseudotuberculosis from milk of camels with multiple abscesses indicates a possible role of milk in transmission of the disease and the economic losses of the malady. Moreover, in cattle, $\mathrm{C}$. pseudotuberculosis was also isolated from milk and from abscesses in various locations, usually intrademal or within lymph nodes ${ }^{[10,11]}$.

In Jordan, information on incidence of camel diseases is scarce. Caseous lymphadenitis has been reported in camels ${ }^{[12]}$. Isolation of $\mathrm{C}$. pseudotuberculosis from pus abscesses, lymph nodes, milk, ticks and blood proved to be due to endemic infection with this organisms. However, the role of imported camels from neighboring countries in transmitting and/ or spreading of such disease in Jordan should be investigated.

The existence of two serotypes of C. pseudotuberculosis isolated from different animal species has been established. Type I was nitrate negative and produced local and general abscesses in guinea pigs, with a prolonged course ( 3 weeks) ending fatally. It included all the strains, originating from sheep and goats, but only three of five cattle strains. Type II comprised all seven isolates from buffaloes and two of the cattle strains. All strains of type II were nitrate positive and did not produce abscesses in guinea pigs, but they caused tissue necrosis at the site of inoculation, with a rapid course (not more that five days) ending in death ${ }^{[7]}$. Furthermore, a recent biochemical and genetic characterization of $\mathrm{C}$. pseudotuberculosis confirmed the existence of two biovars, biovar equi from isolates that reduce nitrate and biovar ovis for isolates that fail to do $\mathrm{so}^{[13]}$. The isolated $C$. pseudotuberculosis strain from camels in the present study was found to belong to type I or biovar ovis (isolated from small ruminants) on the basis of negative nitrate reduction and its virulence to guinea pigs. From the epizootiological point of view, the author suggested that $C$. pseudotuberculosis infection was transmitted to camels in this study from infected sheep and goats because they graze together on the same pasture.

Although in vitro antibiotic susceptibility test showed that C. pseudotuberculosis was fully sensitive to some antibiotics, caseous lymphadentitis may continue to be a serious problem for animal breeders because antibiotics in vivo are unable to penetrate the thick capsule of the abscesses. However, a better understanding of the methods of transmission can be of great help in devising preventive management schemes. Reliable diagnostic tests could be used to identify affected animals for observation, isolation and/or culling.

\section{CONCLUSION}

The results of this study indicates that caseous lymphadenitis was prevalent in dromedary camels in Jordan and the type I strain or biovar ovis is predominant, in spite of the clinical symptoms, nature and distribution of lesions in camels are not as typical as in sheep. Because the infected camels did not response to several broad spectrum antibiotics due the nature of this disease, so the recommended preventive and control measures, application of good sanitary and hygienic measures should include. Adequate control programs in small ruminants may contribute to the reduction in the prevalence of this disease in camels. Also more studies needed to find effective vaccine against this disease in camels and in small ruminants.

\section{REFERENCES}

1. Brown, C.C., H.J. Olander and S.F. Alves, 1987. Synergistic hemolysis-inhibition titers associated with caseous lymphadenitis in a slaughterhouse survey of goats and sheep in northeastern Brazil. Vet. Bull., J. 51: 46-49. http://www.pubmedcentral.nih.gov/articlerender.fc gi? artid=1255272

2. Peel, M.M., G.G. Palmer, A.M. Stacpoole and T.G. Kerr, 1997. Human lymphadenitis due to Corynebacterium Pseudotuberculosis. Clin. Infect. Dis., 24: 185-191. http://cat.inist.fr/?aModele= afficheN\&cpsidt $=2562974$ 
3. Al-Rawashdeh, O.F. and K.M Al-Qudoh, 2000. Effect of Shearing on the incidence of caseous lymphadenitis in Awassi sheep in Jordan. J. Vet. Med., 47: 287-293. http://www.medscape. com/medline/abstract/10861197?prt=true

4. Radwan, A.I., S. El-Magawry, A.D. Hawari, S.I. Al-Bekairi and R.M. Robleza, 1989. Corynebacterium pseudotubercuosis infection in camels in Saudi Arabia. Trop Anim. Hlth Prod., 21: 229-230. http://www.ncbi.nlm.nih.gov/ pubmed/2617677

5. Domenech, J., G. Guidot and D. Les. Richard, 1977. Les maladies pyogènes du dromadaire en Ethiopie. Symptomatologie. Etiologie. Rev. Elev. Med. Vet. Pay. Trop., 30: 251-258. http://publications.cirad.fr/une_notice.php?dk=455 347

6. Afzal, M., M. Sakir and H.M. Majid-Hussain, 1996. Corynebacterium Pseudotuberculosis infection and lymphodenitis (taloa or mala) in the camel. Trop. Anim. Hlth prod., 28: 158-162. DOI: 10.1007/BF02299568

7. Humphreys, F.A. and R.J. Gibbons, Some Observations on Corynebacterium, infection. 1942. Canad. Comp. Med., 6: 35-45. http://www.pubmedcentral.nih.gov/articlerender.fc gi?artid=1584094

8. Brogden, K. A., R.C. Cutlip and H.D. Lehmkuhl, 1984. Experimental corynebacterium pseudotuberculosis infection in lambs. Am. J. Vet Res,45:1532-1534. http://www.ncbi.nlm.nih.gov/pubmed/6476566
9. Tejedor, M.T., J.L. Martin. J.A. Corbera and U. Shulz, 2004. Pseudothuberculosis in dromedary camels in the Canary Islands. Trop. Anim. Hlth. Prod., 36: 459-462. 10.1023/B:TROP.0000035012.63821.12 http://www.springerlink.com/content/18217615433 $82621 /$

10. Kariuki, D.P. and J. Poulton, 1982. Corynebacterium infection of cattle in Kenya. Trop. Anim. Hlth. Prod., 14: 33-36. DOI: 10.1007/BF02281102

11. Hommez, J., L.A. Devriese, M. Vaneechoutte, P. Riegel, P. Butaye and F. Haesebrouck, 1999. Identification of monlipohpilic corynebacteria isolated from dairy cows with mastitis. J. Clin. Microbiol., 37: 954-957 http://cat.inist. fr/?aModele $=$ afficheN\&cpsidt $=1730768$

12. Al-Rawashdeh, O.F., F.K. Al-Ani, L.A. Sharrif, K.M. Al-Qudah, Al- Y. Hami and F. Nicolas, 2000. A survey of camel (camelus dromedarius) Diseases in Jordan. J. Zoo wild. Med., 31: 335-338. DOI: $10.1638 / 1042-$ 7260(2000)031[0335:ASOCCD]2.0.CO,2

13. Songer, J.G., K. Backenback, M.M. Marshall, G.B. Olson and L. Kelley, 1988. Biochemical and genetic characterization of corynebacterium pseudotuberculosis. Am. J. Vet. Res., 49: 223-226. http://www.ncbi.nlm.nih.gov/pubmed/2831763 